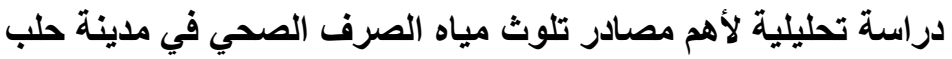

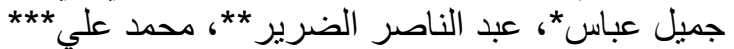

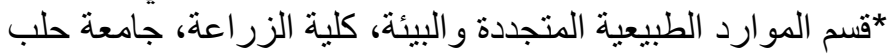

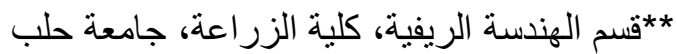

***طالب در اسات عليا (دكتور اه) قسم الموارد الطبيعية المتجدة والبيئة، كلية الزر اعة، جامعة حلب الزبة

\section{الخلاصة}

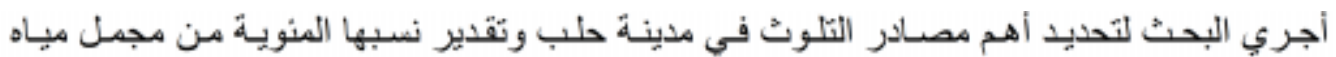

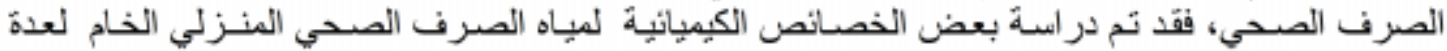

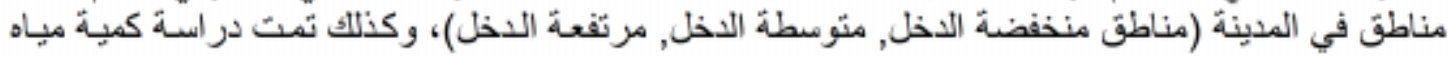
pH, NH

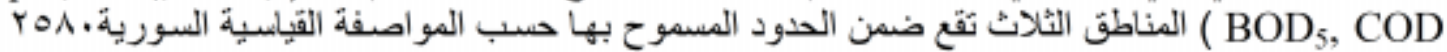

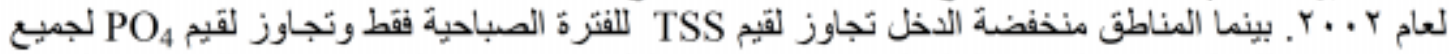

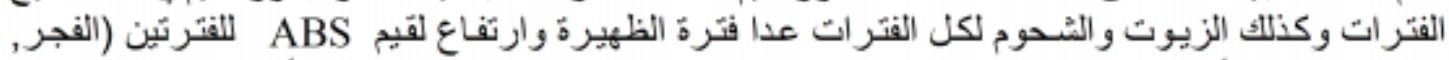

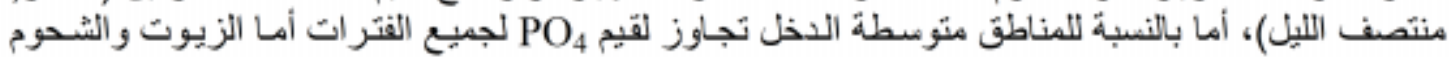

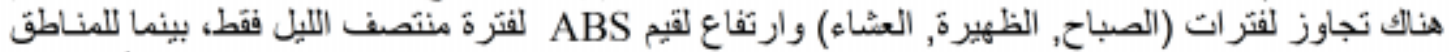

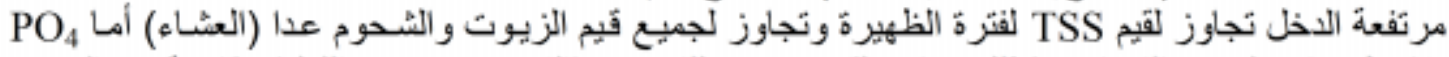

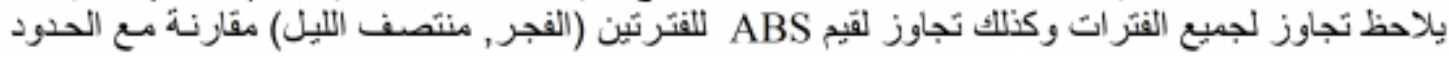

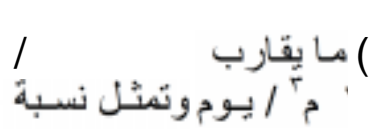

$$
\begin{aligned}
& \text { ها حسب المو اصفة القياسية السورية }
\end{aligned}
$$

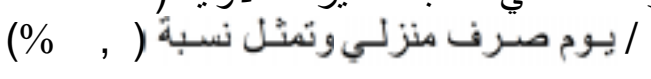

\section{المقدمة}

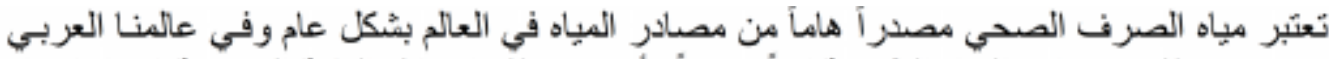

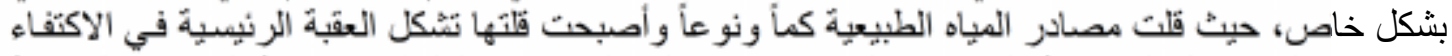

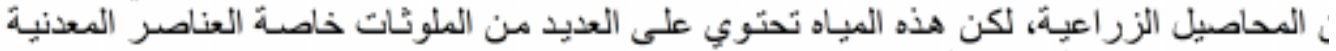

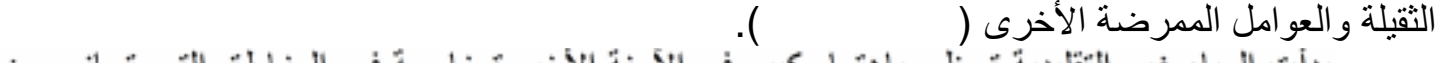

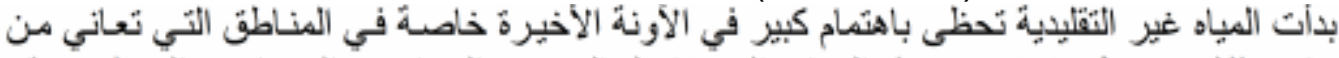

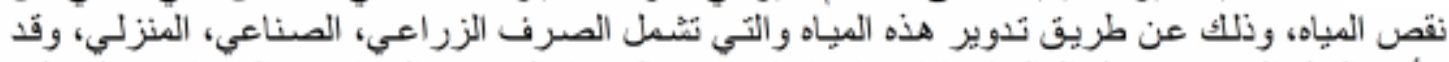

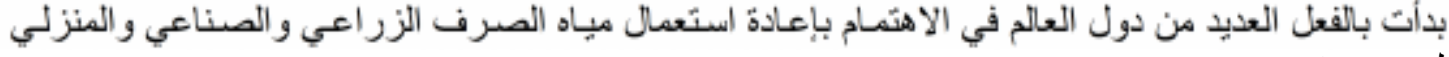

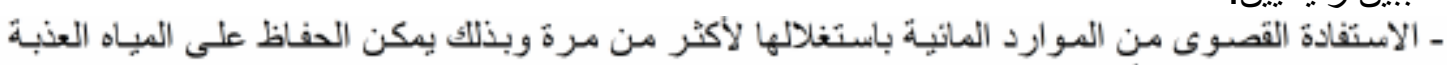

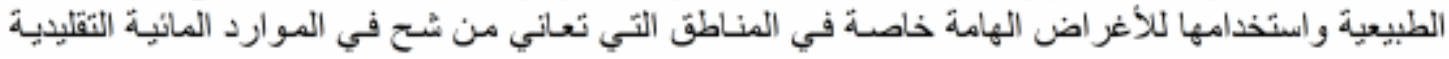

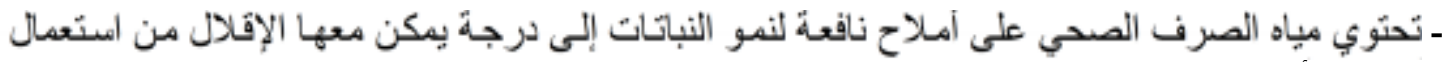

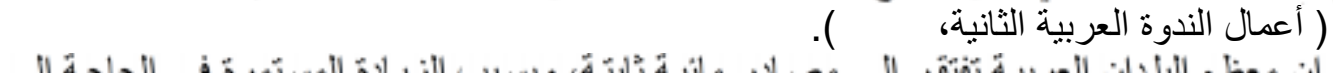

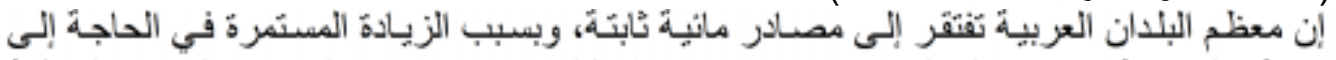

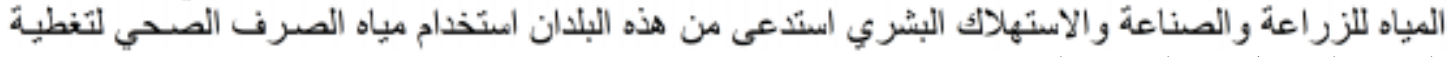

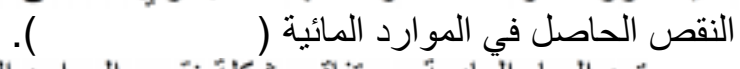

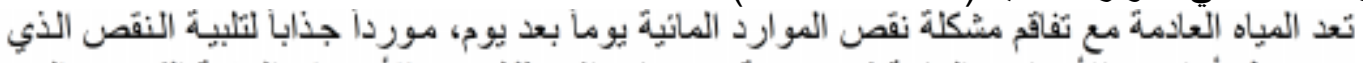

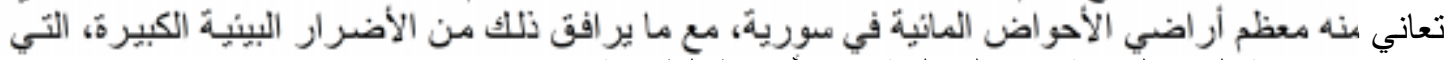

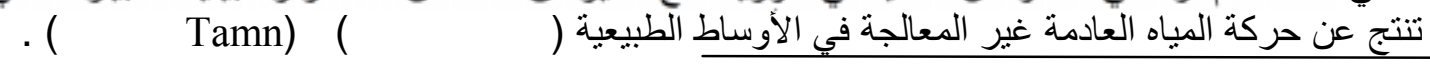

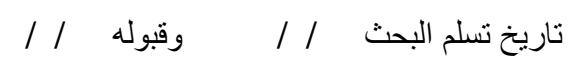




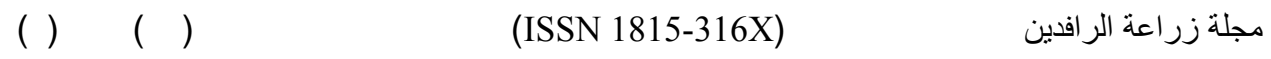

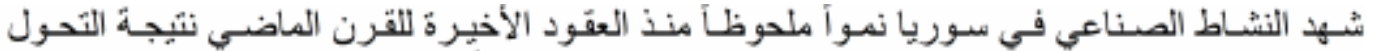

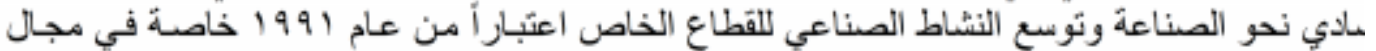

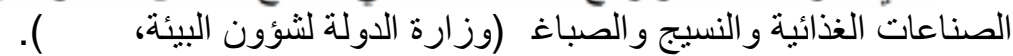

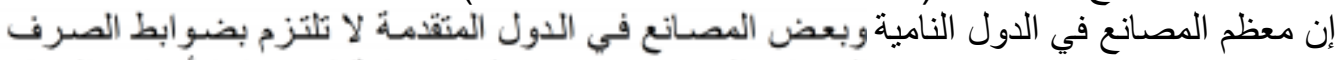

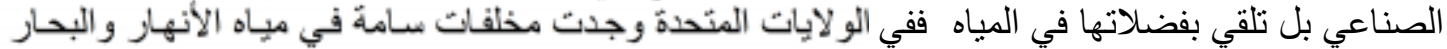

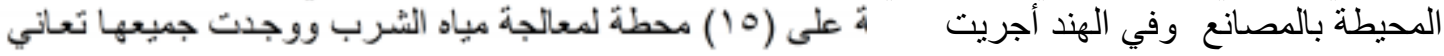

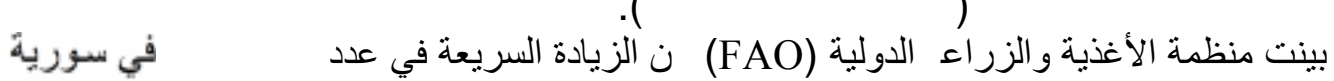

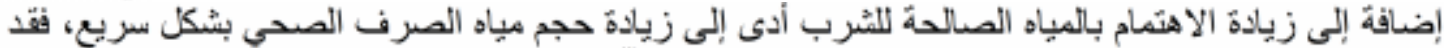

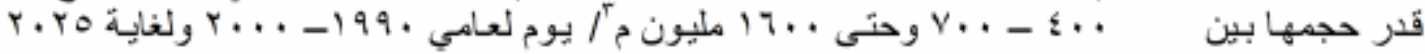
(FAO, )

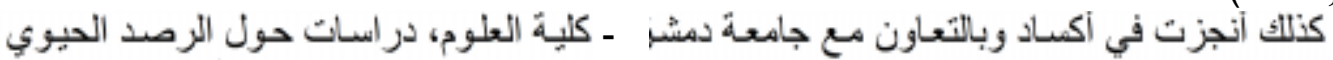

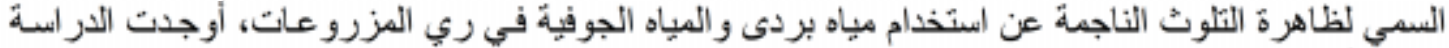

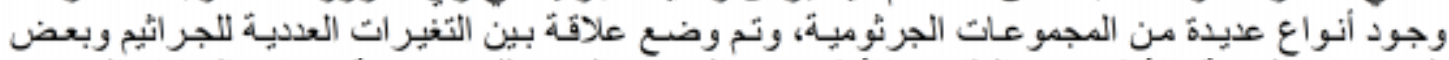

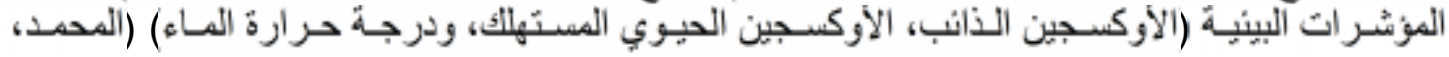

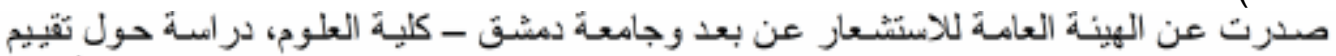

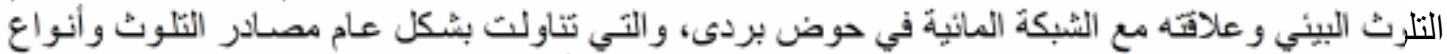

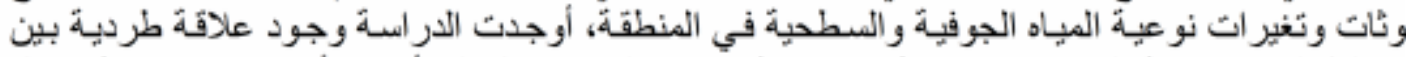

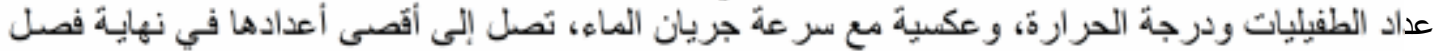

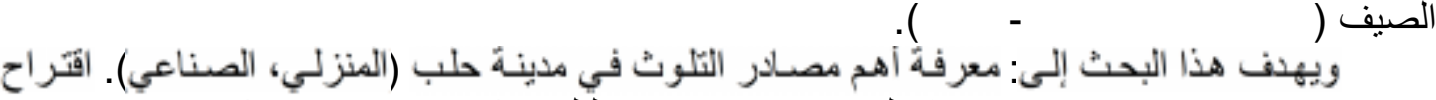

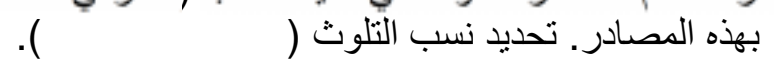

مواد البحث وطرائقه

المواد: الحصول على عينات من مياه

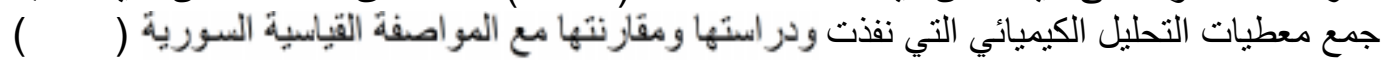

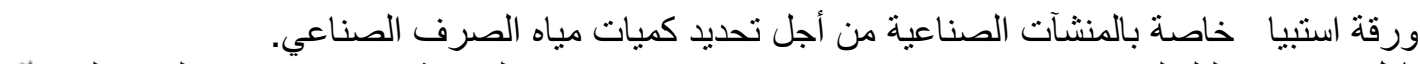

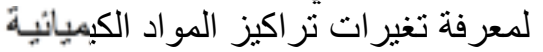

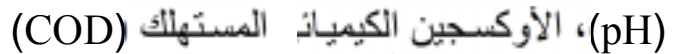

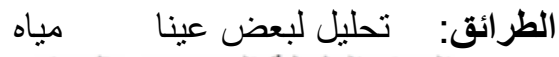
الأوكسجين الحيوي المسنِهلك (BOD

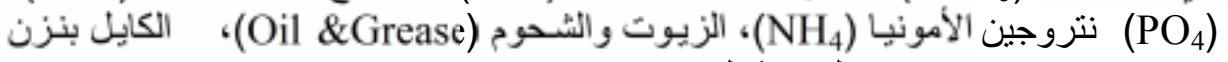
القياسية السوري الزي (ABS)

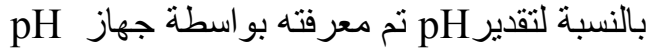

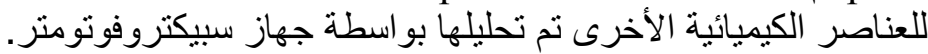

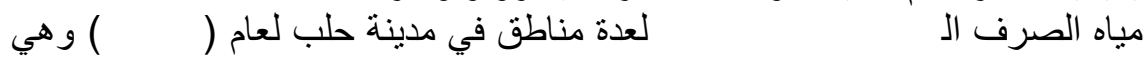
تم تقسيم مناطق الدر اسة حسب نمط المعيشة كالتالي:

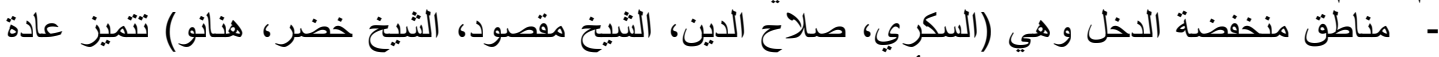

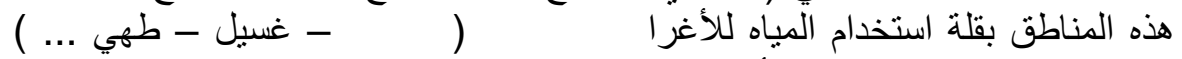

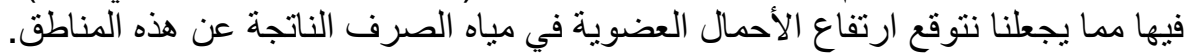

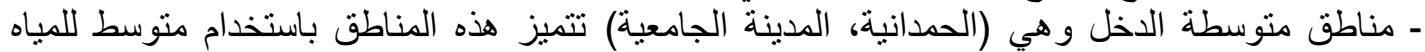

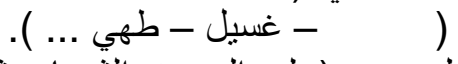

- مناطق مرتفعة الدخل وهي: (حلب الجديدة، الشّهباء، شارع النيل) 

( ) ( )
(ISSN 1815-316X)
لمة زراعة الر افدين

تتميز مناطق مرتفعة الدخل عادة باستخدام كبير للمياه للأغراض المختلفة (استحمام، غسيل، طهي.. )

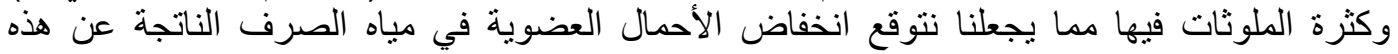

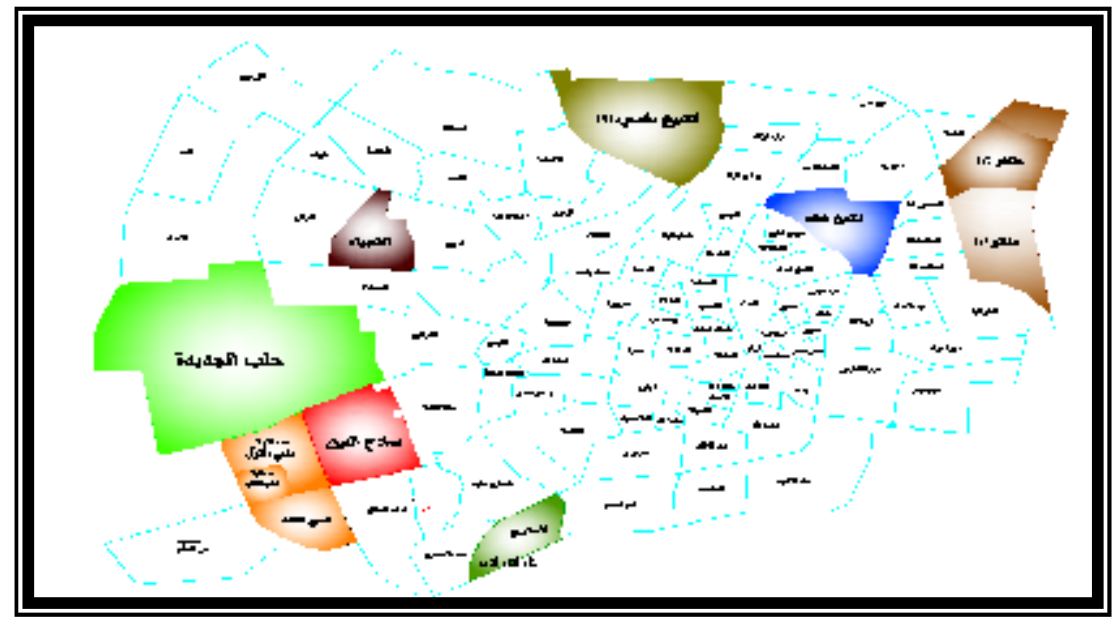

$$
\text { ( ) ( ) يبين المناطق التي تم أخذ العينات منها }
$$

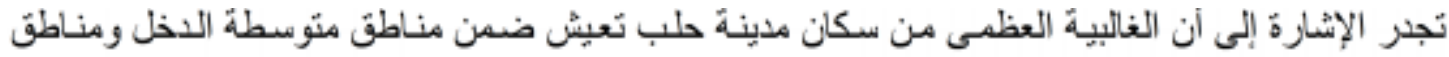

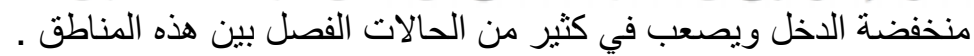

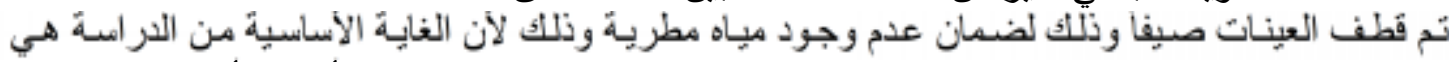
معرفة خصائص مياه الصرف المنزلي الخام (غير المخلوطة بمياه صرف وفن صناعي أو مياه أمطار).

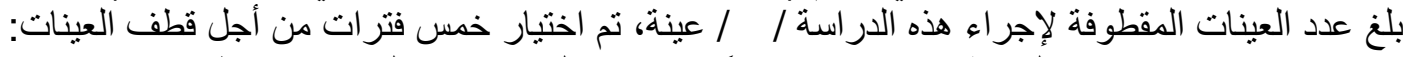

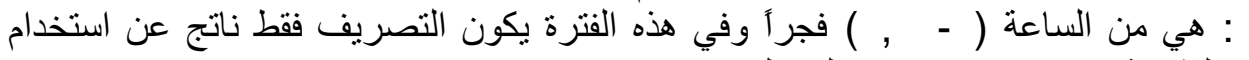

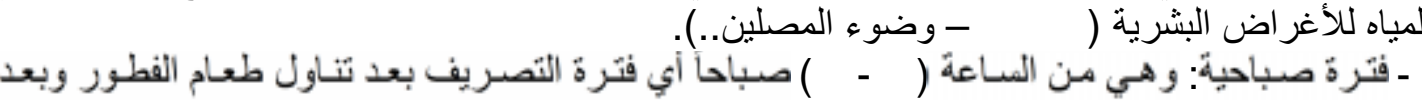

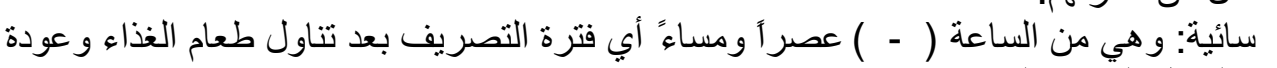
الموظفين و العمال إلى مناز لهم.

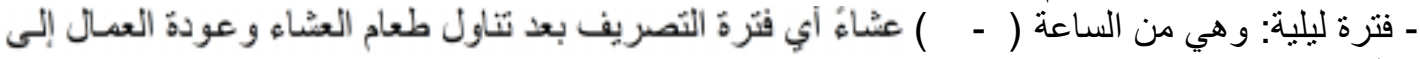
ـ فترة منتصف الليل: هي من الساعة ( - ) ليلا وفي هذه الفترة بكون التصريف فقط نـائج عن استخدام

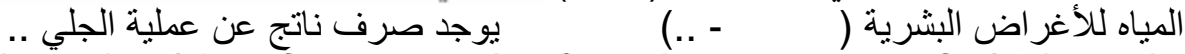

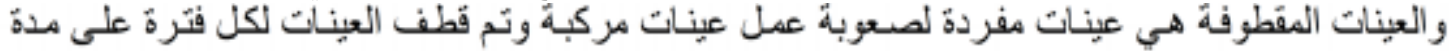

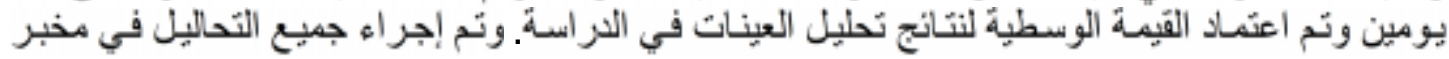

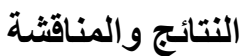

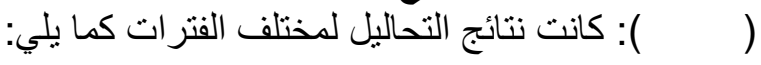

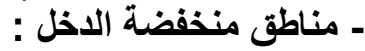

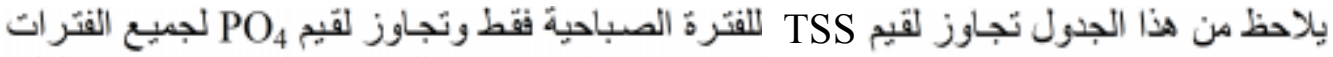

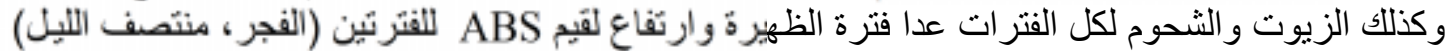

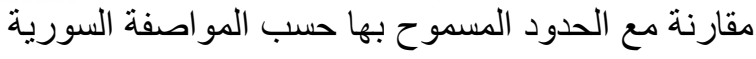
( ) ( ) : نتائج تحاليل لعينات مائية هـ 

( ) ( )
مجلة زر اعة الر افدين

\begin{tabular}{|r|c|c|c|c|r|}
\hline. &. &. &. & $\vee . \leqslant q$ & $\mathrm{pH}$ \\
\hline. &. & &. &. & COD ppm \\
\hline. &. &. &. &. & BOD $_{5} \mathrm{ppm}$ \\
\hline. &. & &. &. & TSS ppm \\
\hline. &. &. &. &. & $\mathrm{TDS}_{\mathrm{ppm}}$ \\
\hline. &. &. &. &. & $\mathrm{PO}_{4} \mathrm{ppm}$ \\
\hline. &. &. &. &. & $\mathrm{NH}_{4} \mathrm{ppm}$ \\
\hline. &. &. &. &. & Oil \& Grease ppm \\
\hline. & - & - & - &. & ABS ppm \\
\hline
\end{tabular}

ـ مناطق متوسطة الاخل :

( ) : نتائج تحاليل لعينات مائية مأخوذة مناطق منوسطة الدخل

\begin{tabular}{|r|r|c|c|c|r|}
\hline & & & & \\
\hline. &. &. &. &. & $\mathrm{pH}$ \\
\hline. &. &. &. & & COD ppm \\
\hline. &. &. &. &. & BOD $_{5} \mathrm{ppm}$ \\
\hline & & &. & & TSS ppm \\
\hline. &. & &. &. & $\mathrm{JDO}_{4} \mathrm{ppm}$ \\
\hline. &. &. &. &. & $\mathrm{NH}_{4} \mathrm{ppm}$ \\
\hline. &. &. &. &. & Oil \& Grease ppm \\
\hline. & &. &. & & ABS ppm \\
\hline
\end{tabular}

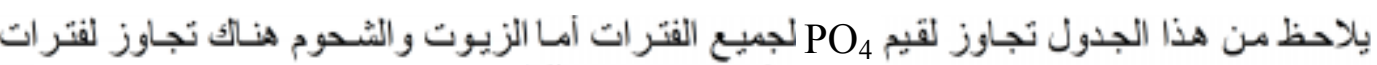

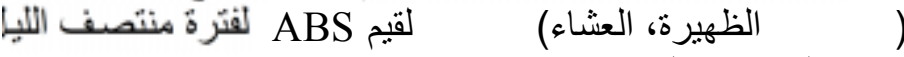

حسب المو اصفة السورية.

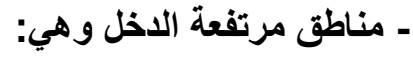

( ) ) نتائج تحاليل لعينات مائية مأخوذة مناطق مرتفعة الدخل

\begin{tabular}{|c|c|c|c|c|c|}
\hline منتصف الليل & & الظهيرة & & & \\
\hline . & . & . & . & . & $\mathrm{pH}$ \\
\hline . & . & & . & . & COD ppm \\
\hline . & & . & . & . & $\mathrm{BOD}_{5} \mathrm{ppm}$ \\
\hline. & . & 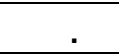 & . & & TSS ppm \\
\hline & . & & . & & TDS ppm \\
\hline . & . & . & . & . & $\mathrm{PO}_{4} \mathrm{ppm}$ \\
\hline . & . & . & . & . & $\mathrm{NH}_{4} \mathrm{ppm}$ \\
\hline . & . & . & . & . & Oil \& Grease ppm \\
\hline . & - & - & - & . & ABS ppm \\
\hline
\end{tabular}

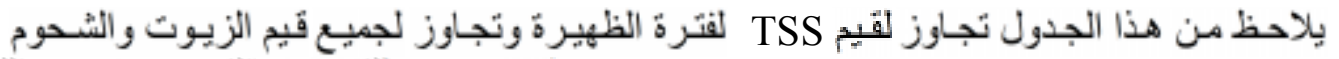

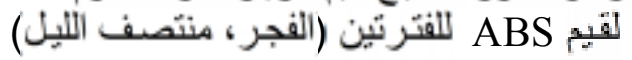
$\mathrm{PO}_{4} \quad$ يلاحظ تجاوز لجميع ها حسب المو اصفة السورية المتوسطات اليومية للمناطق الثلاث وكانت النتائج كما يلي: 

( ) ( )
(ISSN 1815-316X)

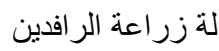

( ): نتائج تحاليل منوسطات يومية لعينات مأخوذة للمناطق

\begin{tabular}{|c|c|c|c|c|}
\hline حسب المو الصفة السورية المسموح به & & & & متوسط يومي \\
\hline. & . & . & . & $\mathrm{pH}$ \\
\hline & . & . & . & COD ppm \\
\hline & s. & . & . & $\mathrm{BOD}_{5} \mathrm{ppm}$ \\
\hline & I & . & . & TSS ppm \\
\hline & 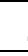 & . & . & TDS ppm \\
\hline & . & . & . & $\mathrm{PO}_{4} \mathrm{ppm}$ \\
\hline & . & - & . & $\mathrm{NH}_{4} \mathrm{ppm}$ \\
\hline & 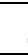 & . & . & Oil \& Grease ppm \\
\hline & . & . & . & ABS ppm \\
\hline
\end{tabular}

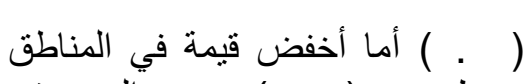

مرتفعة الاخل كمتوسط يومي ( ) ويرجع السبب في في السنا

يلاحظ ما يلي: - ماني

أعلى قيمة (.ppm) (. ppm)

ارتفاعه إلى استخدام المنظفات بكميات كبيرة في تلا

- -

(.)

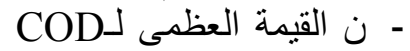

أخفض قيمة في مناطق مرتفعة الدخل لإنل

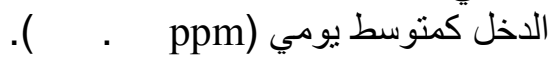

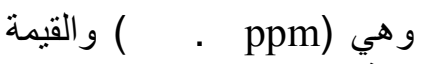

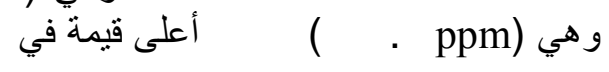

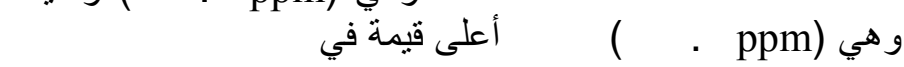
- أن القيمة العظمى

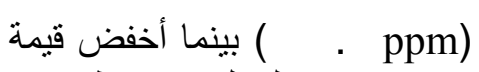

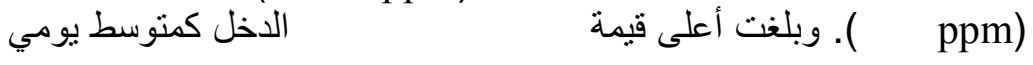
كمتوسط يومي ( . p p
أخفض قيمة في مناطق ( . ppm)

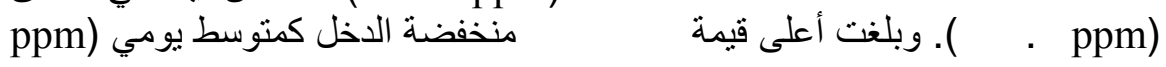

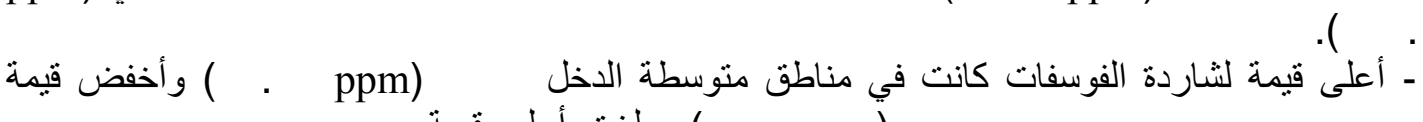
وبلغت أعلى قيمة ( . ppm)

(. ppm)

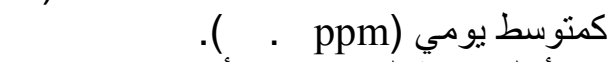

$$
\text { وبلغت أعلى قيمة ( . ppm) }
$$

- أعلى قيمة لنتروجين الأمونيوم

خفض قيمة في ( ppm)

ppm)

$$
\text { وبلغت أعلى قيمة ( ppm) }
$$

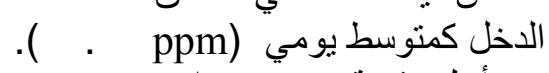

ping) منتصف الليل

$$
\begin{aligned}
& \text { أعلى قيمة } \\
& \text {. ) و أخفض قيمة في مناطق متوسطة لإنة }
\end{aligned}
$$

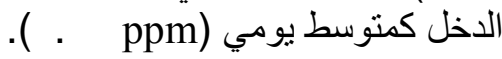

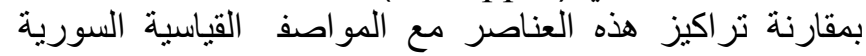

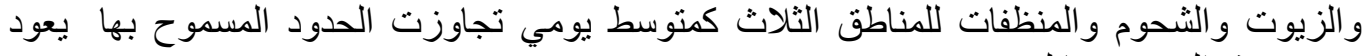

$$
\begin{aligned}
& \text { هذه القيم مايلي: } \\
& \text { - تصريف الزيوت و الثحوم في المنازل بشكل عشو ائي. }
\end{aligned}
$$
- ـ أعلى قيمة للمنظفات (ألكيل بنزن سلفونات) - أعلى قيمة زيوت يوية وا 

( ) ( )
(ISSN 1815-316X)

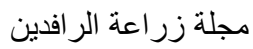

وتجدر الإشارة إلى أن التراكيز المرتفعة من الزيوت والثحوم تلحق ضرراً

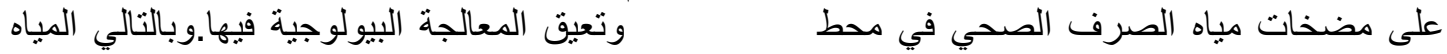
حسب المواصفة السورية غير صالحة للري الزراعي و إنما تحتاج لعمليات معالجة حتى تصبح صالحة لئة

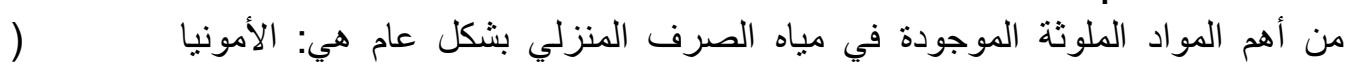

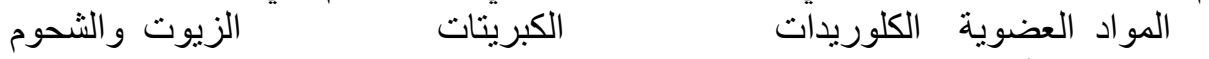

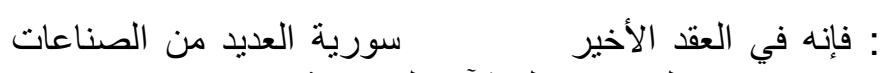

جر اثيم مختلفة

استقطبت مدينة حلب معظ هذه الصناعات تم بناء العديد من المنشآت الصناعية

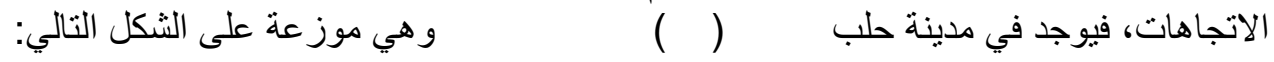

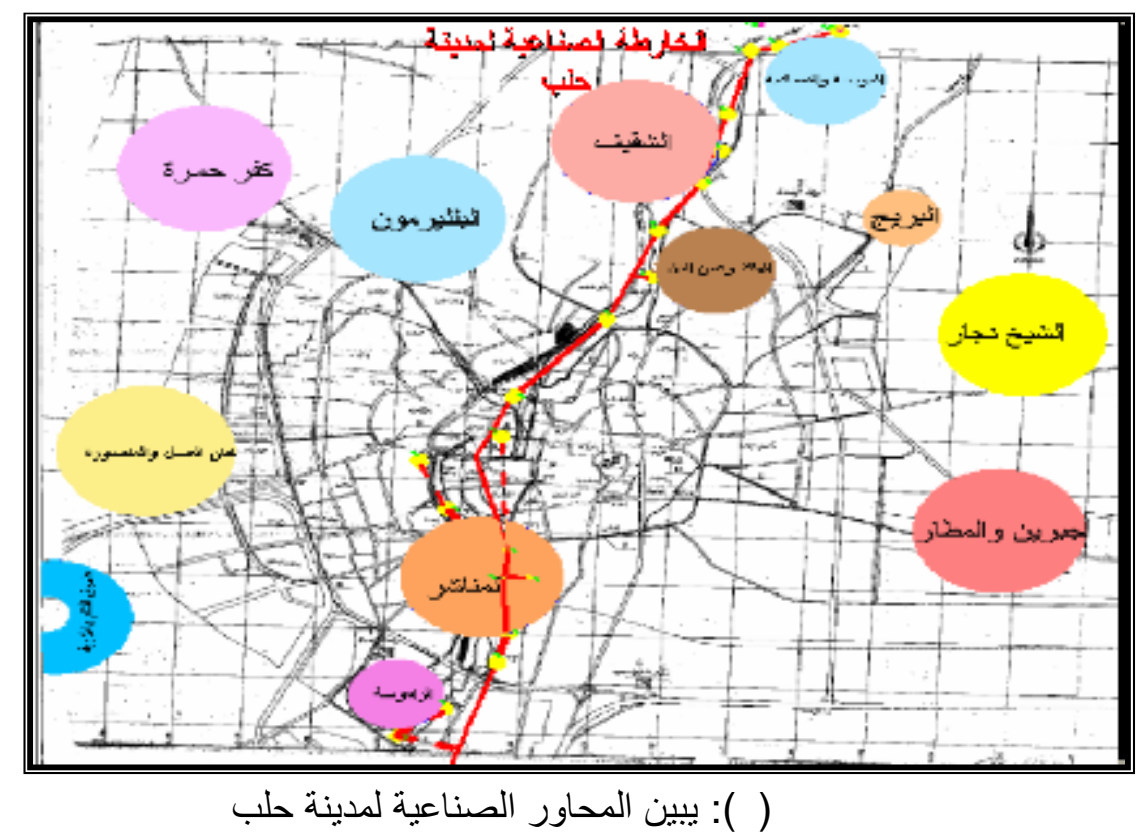

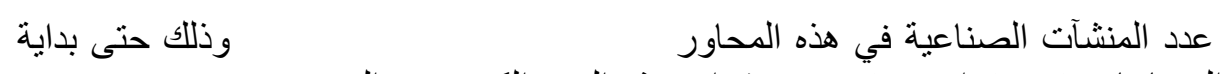

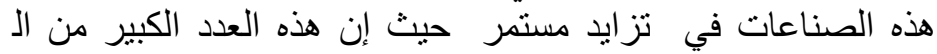

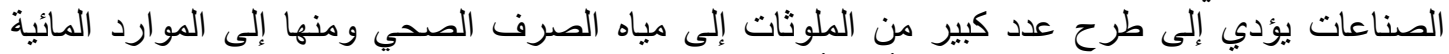

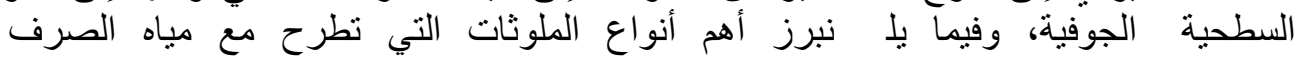

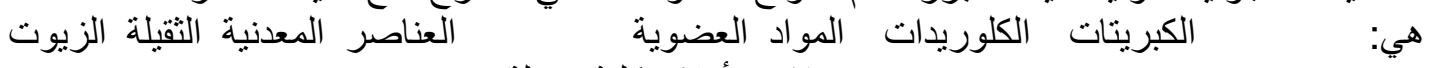

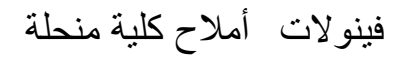

وفيما يلي جدول بعدد المنشآت الصناعية وكميات المياه الصادرة منها:

شآت الصناعية وكميات المياه الصادرة نها لغاية

$:()$

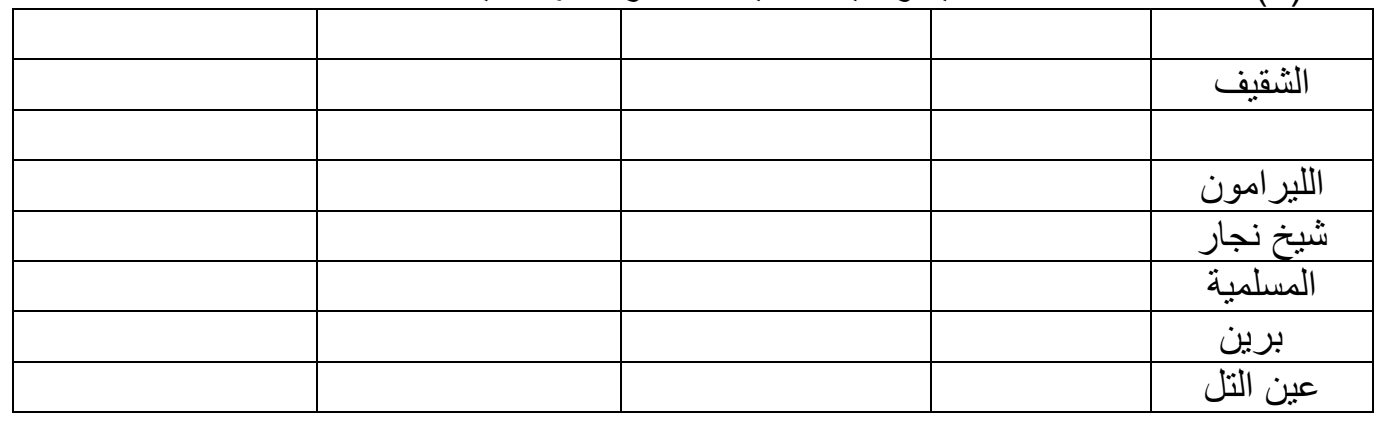



()$\quad(\quad)$

\begin{tabular}{|l|l|l|l|l|}
\hline & & & & البريج|ر \\
\hline & & & & \\
\hline & & & & \\
\hline
\end{tabular}

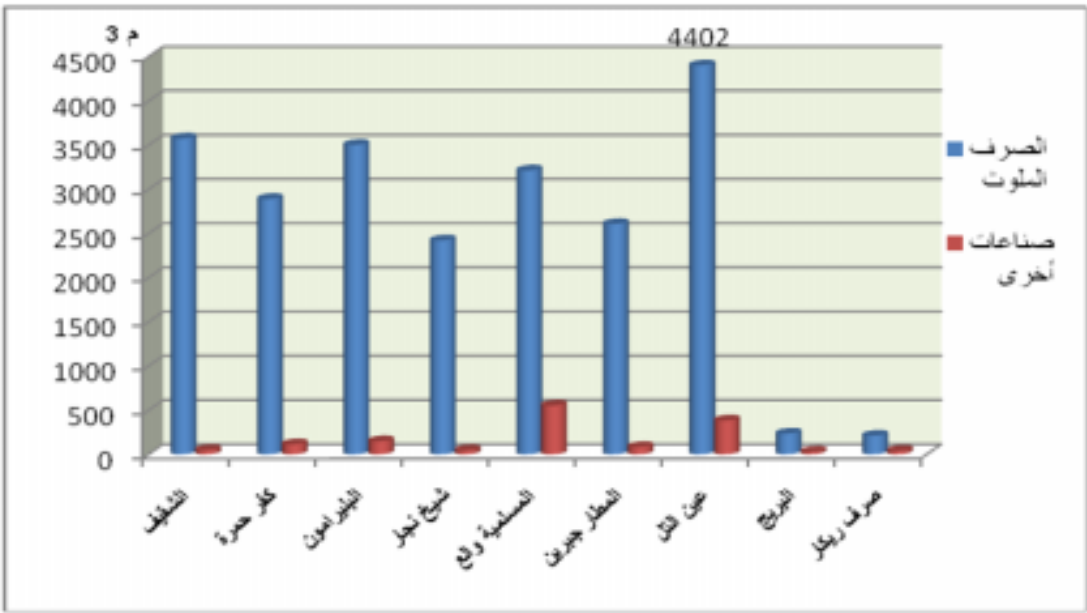

( ) : كمية المياه بين الصناعات ذات الصرف الملوث و والصناعات الأخرى لبداية

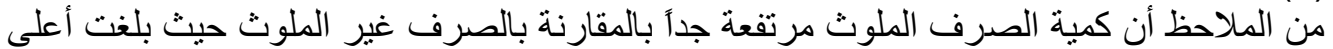

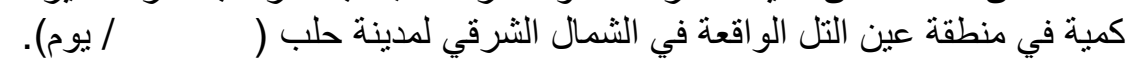

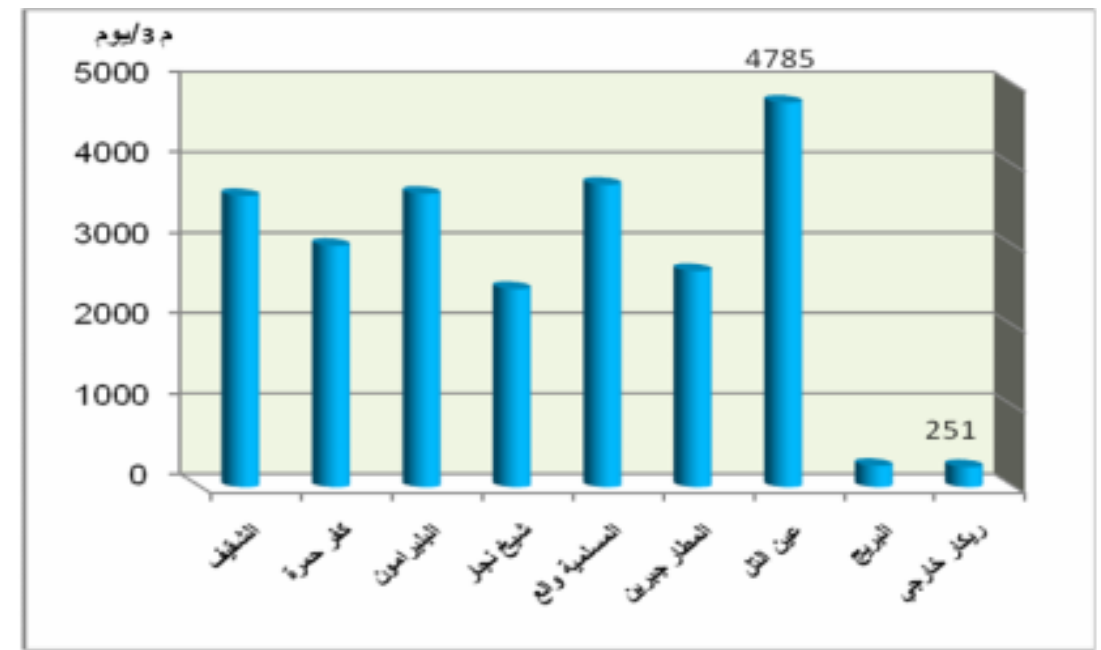

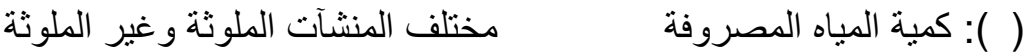

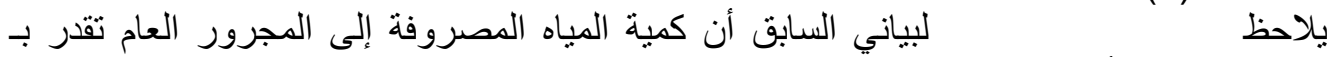

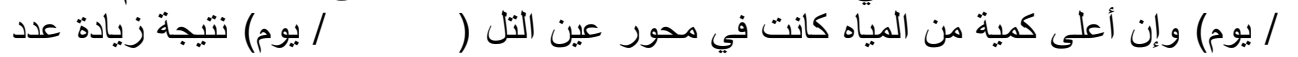

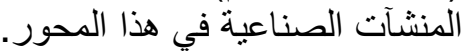

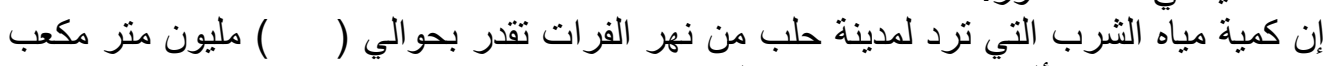

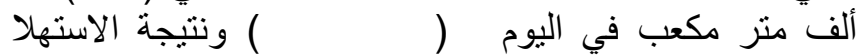

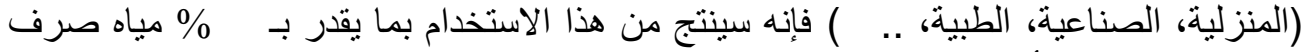

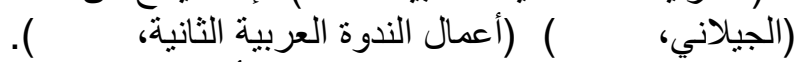

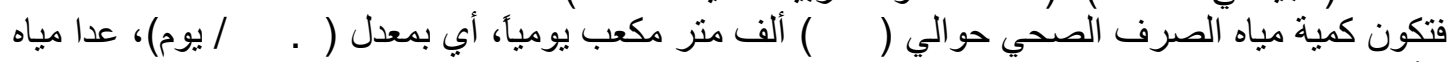

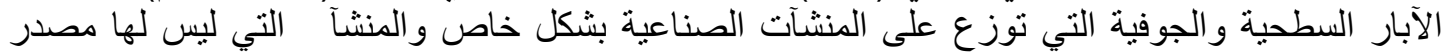




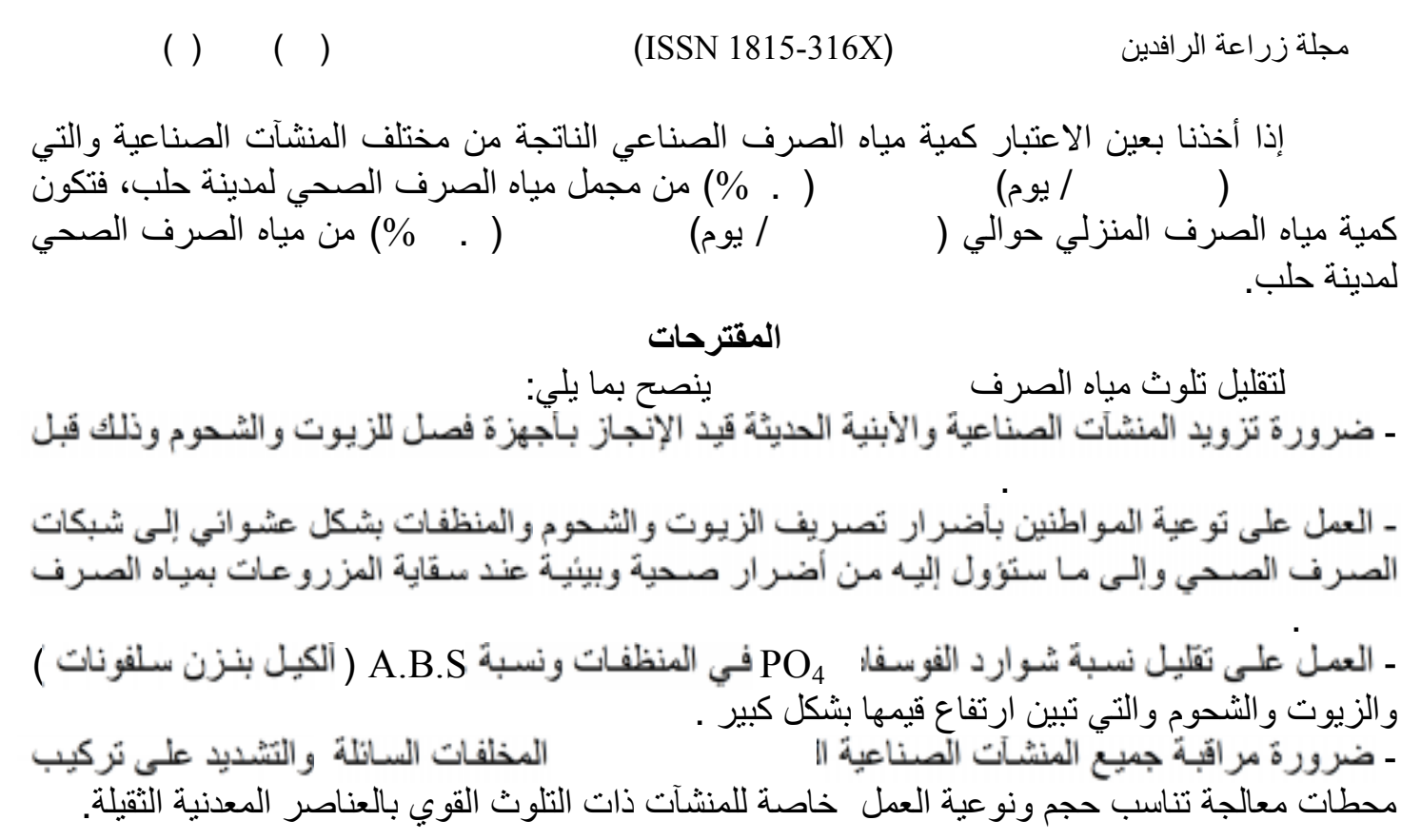

\title{
ANALYTICAL STUDY OF THE MOST IMPORTANT SOURCES OF CONTAMINANTS WASTEWATER IN ALEPPO
}

Jamil Abbass*, Abdulnaser Dharir **, Mohammed Ali ***

*Dept. of Forestry and Ecology, Faculty of Agriculture, University of Aleppo

**Dept. Rural engineering, Faculty of Agriculture, University of Aleppo

***Postgraduate Student (PhD) Dept. of Forestry and Ecology, Faculty of

Agriculture, University of Aleppo

\begin{abstract}
This Study has been conducted to determine the most crucial of pollution and evaluate the percentage portions of these sources out of the whole sewage water in Aleppo city. the chemical characteristics of raw domestic sewage water and the amount of sewage water that goes in to the total run water in some different areas in Aleppo (low, medium, and high income areas).had been studied.For the $\left(\mathrm{pH}, \mathrm{NH}_{4}, \mathrm{TDS}, \mathrm{BOD}_{5}, \mathrm{COD}\right)$, all values are down allowable concentration based Syrian Standards 2850 for 2002. For the low income areas, an excess of TSS values during morning hours. While an excess of $\mathrm{PO}_{4}$ values and those of olis and lubrication have been noticed during different day hours except for the noon time, and an excess of ABS values at dawn and midnights times. For medium in come areas, there has been an excess in the values of $\mathrm{PO}_{4}$ during the different day hours, an excess in the values of oils and lubrications at morning, noon. and night hours. and an excess of ABS values only at midnights. For high in come areas. an excess of TSS values were noticed at noon, an excess of oils and lubrication values at different time except for nights, and an excess of $\mathrm{PO}_{4}$ values at all times and an excess of ABS values at dawn and midnights compared with the allowable concentration based Syrian Standards 2850 for 2002. Based on the initial evaluation, the amount of domestic and industrial sewage water is estimatedat $480000 \mathrm{~m}^{3} /$ day. Out of this amount about $455500 \mathrm{~m}^{3} /$ day that is $94.9 \%$ comes form domestic consumption, and 24500 $\mathrm{m}^{3} /$ day $(5.1 \%)$ is industrial sewage.
\end{abstract}



()$\quad(\quad)$
(ISSN 1815-316X)

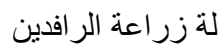

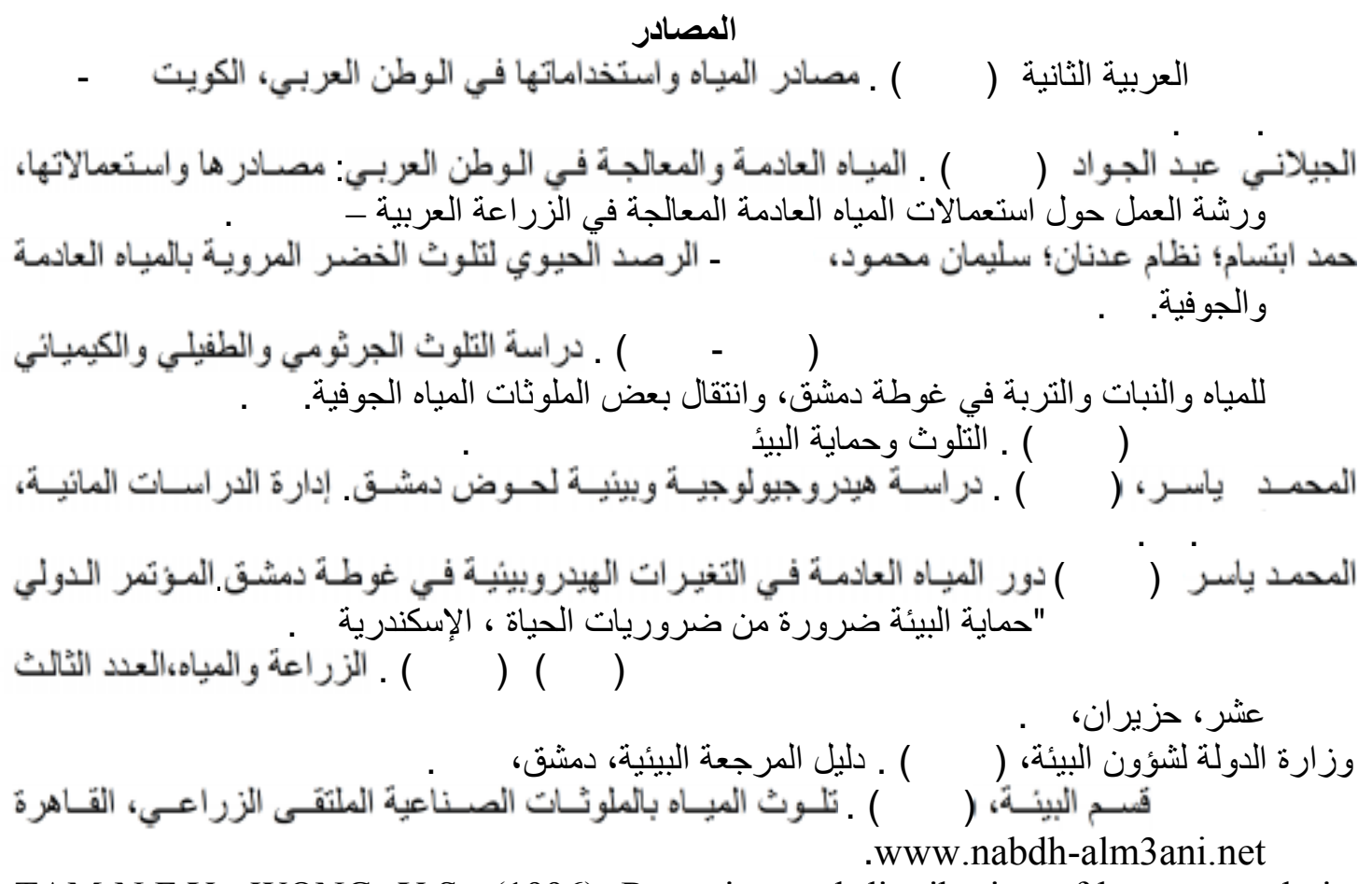

TAM N.F.Y.; WONG. Y.S., (1996). Retention and distribution of heavy metals in mangrove soils receiving waste water. Environment Pollut, 94, 283-291.

FAO, (1997b). Seawater Intrusion in Coastal Aquifers: Guidelines for study, Monitoring and Control. FAO, Rome, Italy.

http:// www.bocome.com .(2008) . 